\title{
Impact of Binder on AISI 316L Microcomponents Produced by Hot Embossing: SEM/EBSD Analysis
}

\author{
E. W. Sequeiros ${ }^{1}$, R. F. Santos ${ }^{2}$, M. T. Vieira ${ }^{2}$ and M. F. Vieira ${ }^{1}$ \\ 1. CEMUC, Department of Metallurgical and Materials Engineering, University of Porto, Rua Doutor Roberto \\ Frias s/n, 4200-465 Porto, Portugal. \\ 2. CEMUC, Department of Mechanical Engineering, University of Coimbra, Pinhal de Marrocos 3030-788 \\ Coimbra, Portugal.
}

Microfabrication technologies not only possess a considerable market potential but are also regarded as promising technologies for today and for the near future, due to the growing demand for microengineering applications such as micromolds, micromechanical structures, sensors and micromedical devices [1]. Metal powder hot embossing is a new micro-manufacturing alternative to conventional powder metallurgy processes, providing dimensional precision and micro details in metallic components [2,3]. This new powder metallurgy approach requires four distinctive steps: (1) preparation of feedstocks (selection and characterization of powder and binder); (2) shapeforming of the component by hot embossing (green part); (3) debinding (brown part); and (4) sintering (final part). These steps are interrelated and the variables of each one affect the properties of final products.

Two different feedstocks were tested, prepared with $316 \mathrm{~L}$ stainless steel powder $(\mathrm{d} 50=3.63 \mu \mathrm{m})$ and two different commercial binders (Licomont and M1, delivered by Clariant ${ }^{\circledR}$ and Atect ${ }^{\circledR}$, respectively). The powder/binder volume ratio was $60 / 40$. The embossing step was done under uniaxial pressure, using a mold with a silicon rubber die, inside an infrared heater chamber. Embossing conditions were studied and optimized in previous work (table 1). Debinding was performed in argon $+5 \% \mathrm{H}_{2}$ atmosphere at a pressure of $5 \mathrm{~Pa}$. To evaluate the replication of micro details, surface characterization was done by stereomicroscopy, scanning electron microscopy (SEM) and 3D micro measurements. Microstructural characterization was done by optical microscopy (OM) and electron backscattered diffraction (EBSD).

Comparing both binders, Licomont allows a better replication of micro details, especially visible in the green parts (Fig. 1(a)), but with M1 a better superficial quality was achieved (Fig. 1(b)). Similar microstructures were observed with both binders: equiaxed austenite grains and some porosity (Fig. 2). Inverse pole figures (Fig. 3) show similar average grain size for both conditions tested, 26 and $30 \mu \mathrm{m}$, for Licomont and M1, respectively. The grain size is almost ten times higher than the initial powder size, which shows that sintering step was effective.

In conclusion the production of micro-detailed metallic parts with both binders is viable; the binders only have impact in embossing and debinding steps. Micro hot embossing of metal powder could become an interesting replicative process, as it has become strategic and crucial to produce dimensional precision and micro-detailed metallic parts/devices, associated with low cost production [5].

References:

[1] M. Worgull., in Micro \& Nano Technologies, ed. J. Ramsden (Elsevier: William Andrew, UK).

[2] M. Sahli, J. C. Gelin and T. Barriere, Powder Technology 246 (2013) p. 284.

[3] E.W. Sequeiros et al.; Euro PM2013 Congress \& Exhibition Proceedings 3 (2013) p. 201.

[4] E. W. Sequeiros, "Micromanufacturing of metallic components by hot embossing"; PhD thesis, Universidade do Porto (2014) (in portuguese).

[5] The authors acknowledge to Fundação para a Ciência e Tecnologia (FCT) through the doctoral grant SFRH/BD/61840/2009 and Tooling EDGE. This research is sponsored by FEDER funds through the program COMPETE - Programa Operacional Factores de Competitividade - and by national funds through FCT, under the project PEst-C/EME/UI0285/2013. 
Table 1. Embossing conditions [4].

\begin{tabular}{|c|c|c|c|}
\hline Feedstock & Pressure (MPa) & Temperature $\left({ }^{\mathbf{}} \mathbf{C}\right)$ & Time (min) \\
\hline C_M1 & 8 & 230 & 30 \\
\hline C_Licomont & 11 & 180 & 30 \\
\hline
\end{tabular}

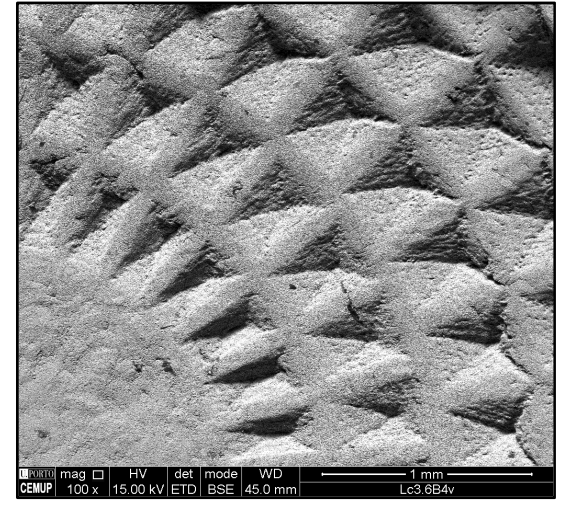

(a)

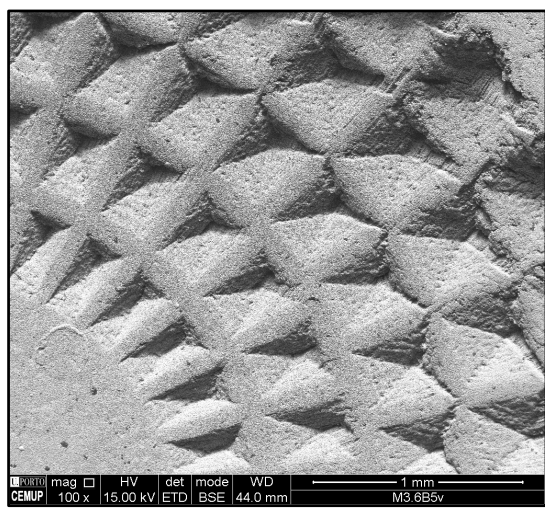

(b)

Figure 1. SEM images of green parts with $C_{-}$Licomont (a) and C_M1 (b).

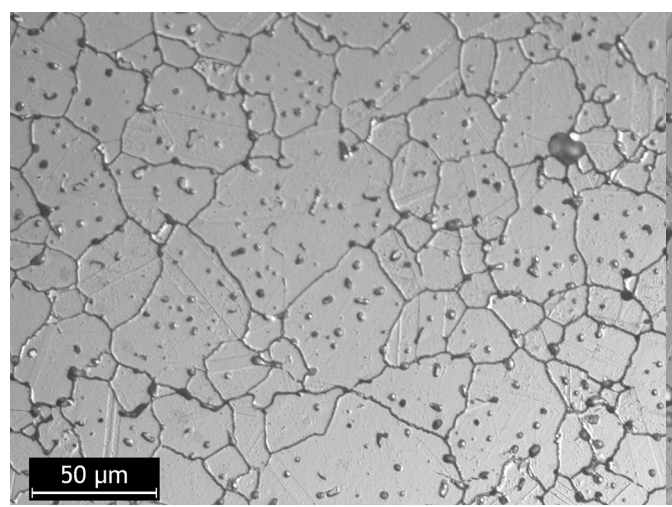

(a)
$50 \mu \mathrm{m}$

(b)

Figure 2. OM images of final parts with C_Licomont (a) and C_M1 (b).

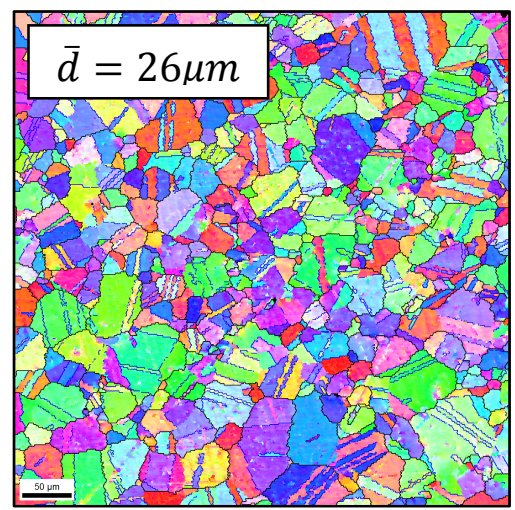

(a)

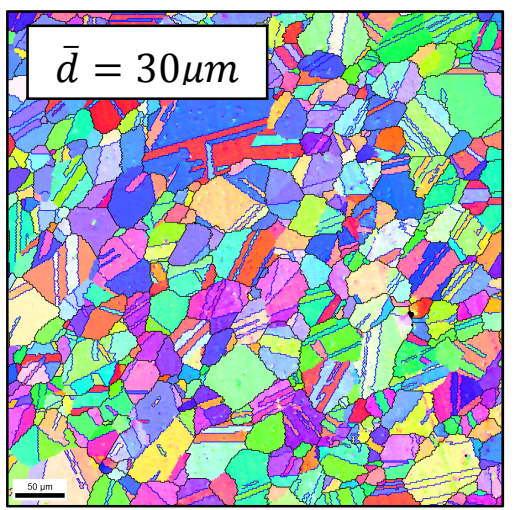

(b)

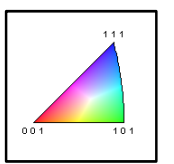

Figure 3. Results of EBSD performed at the final parts with C_M1 (a) and C_Licomont (b) 